\section{Getting ghrelin's GOAT}

\section{By Steve Edelson, Executive Editor}

When the discovery of ghrelin was reported in 1999, the appetitestimulating hormone was hotly pursued by biotech and pharma companies, with agonists aimed at treating various wasting syndromes and antagonists geared toward treating obesity. ${ }^{1}$ However, a number of ghrelin modulators have since fallen off the map because of unwanted side effects or a lack of efficacy.

Companies continuing to pursue ghrelin suspect that the stumbles of their peers were compound specific. Now, researchers at the University of Texas Southwestern Medical Center and colleagues have identified the enzyme that activates ghrelin, thus providing a target for indirectly modulating the hormone.

Whether indirect modification will lead to improved therapeutics compared to direct ghrelin agonists or antagonists of the G protein-coupled receptor (GPCR) for ghrelin is unknown, but pursuing an enzyme rather than a GPCR could allow for better structurebased drug design.

Ghrelin is secreted in the stomach and stimulates appetite. In humans, ghrelin levels increase in response to fasting and decrease after meals. ${ }^{2}$ Ghrelin also promotes gastric motility and stimulates the release of growth hormone from the pituitary gland.

For ghrelin to become active, the hormone needs to be acylated with octanoate, which is an eight-carbon fatty acid. The enzyme responsible for the $O$-acylation, however, was unknown. Now, in a paper in Cell, the UT Southwestern researchers identified ghrelin $O$-acyltransferase (GOAT) as the enzyme that attaches octanoate to ghrelin. ${ }^{3}$

The group looked at the family of acyltransferases that catalyze $\mathrm{O}$-acylation reactions. Through studies of the mouse genome, the researchers showed that 16 such enzymes exist in mice, and a series of molecular biology experiments narrowed the list down to a single enzyme, dubbed GOAT, responsible for octanoylation of ghrelin.

With the enzyme in hand, the authors wrote that "the discovery of GOAT opens the way to a search for chemical inhibitors that may be useful in controlling appetite."

The group also noted that a GOAT inhibitor should not have off-target activity because "no other protein is known to be octanoylated."
"This is a long sought-after enzymatic activity and presents itself as another approach to modulate ghrelin," said Peter DiStefano, CSO of Elixir Pharmaceuticals Inc., which is developing ghrelin agonists and antagonists.

For companies developing ghrelin modulators, an unanswered question is: what happens in mice when GOAT is knocked out?

"You'd need to prove that interfering with the enzyme has the desired physiological effect on food intake," said Mark Peterson, VP of IP and operations at Tranzyme Pharma Inc., which is also developing compounds that agonize and antagonize ghrelin. "Generally there's not an absolute requirement for acylation, and even without GOAT there's likely to be effects of ghrelin," as the nonacylated form of the molecule still could have physiological effects.

DiStefano agreed: "The $\$ 64,000$ question is: if I take the enzyme away, do I get the same metabolic profile as when I knock out or antagonize the receptor? A GOAT knockout won't be able to make active ghrelin, but there's a school of thought that nonactivated ghrelin still has an effect. I'm going to guess that the GOAT knockout mouse phenotype is going to be very interesting."

The UT Southwestern researchers declined to discuss their next steps in studying GOAT.

Peterson noted that because GOAT is an enzyme, it should be much easier to crystalize than the ghrelin receptor. "An enzyme is beautiful for doing structure-based design," he said. "Lots of tools can be brought to bear. When you deal with a GPCR, you don't have as many tools for drug discovery." Indeed, only two GPCRs have ever been crystalized: rhodopsin and adrenergic receptor $\beta 2$.

On the other hand, said Tranzyme president and CEO Vipin Garg, GOAT is "one step removed from getting at ghrelin's activity. Not going directly after the receptor introduces additional risk."

Tranzyme's TZP-301, a small-molecule ghrelin antagonist and inverse agonist, is in preclinical development.

The company is hoping its hybrid approach to blocking ghrelin will lead to better results than those of other companies' ghrelin antagonists. "Ghrelin is constitutively active," noted Peterson. "Generally what you want in those cases is to have an agent that actually brings you below the baseline activity of the receptor, rather than just suppressing the activity" with a pure antagonist. Inverse agonists essentially lock a given receptor in an inactive state.

Ghrelin antagonists have hit stumbling blocks in the clinic. In 2006, Cytos Biotechnology AG halted development of its CYT009-GhrQb immunodrug after it failed to cause significant weight loss compared with placebo in a Phase I/IIa trial in 111 obese patients. Cytos said the agent, which induced antighrelin antibodies, was the first clinical trial of a ghrelin antagonist. ${ }^{4}$

"There's been a parade of pharmaceutical problems with the ghrelin antagonists that are out there," said Elixir's DiStefano. "Other groups 


\section{TARGETS \& MECHANISMS}

have had problems with oral bioavailability and toxicity."

In addition to those issues, DiStefano thinks obesity "is not the therapeutic area you should look at for ghrelin antagonists-it's diabetes. Ghrelin stimulates fat deposition. In our knockout mice, blocking ghrelin does not decrease food intake, but it does prevent the animals from gaining fat. Body weight does lower, but it's not dramatic. But we do see increased insulin sensitivity. That's driven us away from obesity and toward type 2 diabetes."

Elixir hopes to start clinical studies of a ghrelin antagonist to treat type 2 diabetes in the fourth quarter.

The company also aims to begin human testing of a ghrelin agonist this year. EX1314, in-licensed from Bristol-Myers Squibb Co., is expected to enter the clinic this year for opioid-induced bowel dysfunction and cancer cachexia.

Tranzyme also has ghrelin agonists in the clinic. Its TZP-101 intravenous agonist is in Phase II testing for postoperative ileus (POI), while an oral agonist, TZP-102, is in Phase II for mild to moderate gastroparesis. Garg said data from the POI trial are expected in May or June, with the gastroparesis data expected in July.

\section{REFERENCES}

1. Kojima, M. et al. Nature 402, 656-660 (1999)

2. Cummings, D.E. et al. Diabetes 50, 1714-1719 (2001)

3. Yang, J. et al. Cell; published online Feb. 8, 2008; doi:10.1016/ j.cell.2008.01.017

Contact: Michael Brown, University of Texas Southwestern Medical Center, Dallas, Texas

e-mail: mike.brown@utsouthwestern.edu

Contact: Joseph Goldstein, same affiliation as above

e-mail: joe.goldstein@utsouthwestern.edu

4. Edelson, S. BioCentury 14(50), A14; Nov. 13, 2006

COMPANIES AND INSTITUTIONS MENTIONED

Bristol-Myers Squibb Co. (NYSE:BMY), New York, N.Y. Cytos Biotechnology AG (SWX:CYTN), Zurich, Switzerland Elixir Pharmaceuticals Inc., Cambridge, Mass.

Tranzyme Pharma Inc., Research Triangle Park, N.C.

University of Texas Southwestern Medical Center, Dallas, Texas 